\title{
How Does the Mind Handle Uncertainty in Ambiguous Figures?*
}

\author{
Manuel Moreno-Sanchez \\ University of Barcelona, \\ Barcelona, Spain
}

\author{
J. Antonio Aznar-Casanova \\ Institute for Brain, Cognition \& \\ Behavior (IR3C), University of \\ Barcelona, Barcelona, Spain
}

\author{
Nelson Torro-Alves \\ Federal University of Paraíba, \\ Paraíba, Brazil
}

\begin{abstract}
How does the mind select one interpretation from a bistable stimulus and how this eventually becomes conscious? We briefly presented 17 rotations of an ambiguous figure to observers and asked them to give a quick response. We were interested in determining how observer factors, stimulus properties and context influence the selected response. Data analysis revealed that observers assigned probabilistically each figure rotation to a category according to an implicit criterion of typicality or prototype. From discriminant analyses we ascertain how the standardized coefficients change as do the testing conditions, mainly when stimulus information is lacking or confusing. Results suggest that the proximity to the prototype expresses the uncertainty of the subject's response and may be gradually manipulated by the orientation of the figure. Depending on the uncertainty value, discriminant strength of the observer and contextual factors have greater influence on responses than the physical properties of the ambiguous stimulus.
\end{abstract}

Keywords: bistable visual perception, ambiguous figures, uncertainty and information, categorization, visual awareness

\section{Introduction}

Ambiguous figures have attracted the interest of the scientific community because they can be used to investigate potential dissociations between stimulus-driven (sensory) and conceptually-driven (cognitive) processes on a neural basis (Kornmeier \& Bach, 2004, 2012; Leopold \& Logothetis, 1999; Zeki, 2004). When observers fixate on bistable ambiguous figures, perceptual alternations can take place, meaning that either one or the other interpretation is selected by visual awareness (Attneave, 1971; Kornmeier \& Bach, 2012; Long \& Toppino, 2004, for a review). In the four ambiguous figures shown in Figure 1, perceptual bistability implies giving a specific meaning to a particular feature. Thus, as pointed by early research (e.g., Flügel, 1913; Boring, 1942), the perceived interpretation of the figure depends on the set of features receiving primary processing (the focal-feature hypothesis). In this vein, a great deal of experimental work has demonstrated that eye movements, such as saccades (Ellis \& Stark, 1978; García-Pérez, 1989), are related to perceptual switching. However, there is also strong evidence to suggest that eye movements are not required for figure reversal (Gale

\footnotetext{
*Acknowledgements: This work was funded through a grant awarded by the Spanish Ministry of Economy and Competitiveness (MINECO) to J. A. Aznar-Casanova (reference PSI2012-35194).

Manuel Moreno-Sanchez, MSSc., Department of Basic Psychology, University of Barcelona.

J. Antonio Aznar-Casanova, Ph.D., Institute for Brain, Cognition \& Behavior (IR3C), University of Barcelona.

Nelson Torro-Alves, Ph.D., Department of Psychology, Federal University of Paraíba.
} 
\& Findlay, 1983; Gregory, 1970), but rather that the direction of attention is the critical factor (Posner, 1980; Tsal, 1994). Central cortical structures have long been invoked (e.g., Hering or Helmholtz) to explain perceptual reversal. Ambiguous figures demonstrate the role of expectations, world-knowledge and the direction of attention (see Long \& Toppino, 2004) in relation to the perceptual interpretation of the stimulus.

One of the basic assumptions in research on perceptual switching with bistable figures is that the two perceptual states underlying a particular conscious interpretation must be based on the internal neural activity in the brain (Kornmeier \& Bach, 2012). This activity can be either endogenously or exogenously induced. However, the question that remains to be answered is how the observer decides upon the selected response. Ascertaining the weight of certain factors that determine some sort of interpretation in bistable figures and identifying the conditions under which they may occur could help to understand how the interplay between physical properties and mental states leads to visual awareness.

Psychophysical and physiological evidence from the neural channel selectively tuned to certain properties (e.g., spatial frequency or orientation) has been used in an attempt to explain phenomenal reversal with ambiguous figures in terms of satiation or fatigue of low-level mechanisms, in other words, as the result of neural adaptation (Babich \& Standing, 1981; Blakemore \& Campbell, 1969; De Valois, 1977; Hochberg, 1950; Maffei, Fiorentini, \& Bisti, 1973). However, if the ambiguous figure is presented briefly, neither adaptation nor satiation processes can be prevented. Whatever the case, this passive bottom-up "neural-channel" model has been criticized by researchers (Bruner \& Leigh, 1955; Bugelski \& Alampay, 1961; Rock, Hall, \& Davis, 1994; Toppino, 2003) in favour of another explanation based on more active, top-down or cognitive processes (e.g., learning, decision making, attention, familiarity or knowledge, expectations, etc.).

To summarize, previous studies (Bugelski \& Alampay, 1961; Goolkasian, 1987; Rock \& Mitchener, 1992) have identified several factors that affect the observer's response to ambiguous figures. These factors can be classified into three groups: (1) observer-related factors, such as previous experience, the criterion of responses in tests, ability to discriminate properties, etc.; (2) stimulus-related factors, such as size (i.e., spatial frequencies selected by filtering the image), complexity, the orientation of the figure, etc. or viewing conditions; and (3) the context in which the stimuli are inserted or the attitudes (expectations) of the observer induced by a set of instructions capable of changing his or her mind. What is unclear is how much each factor contributes to the observer's response and how these factors interact with one another. Do these factors explain how the interpretation of an ambiguous figure is selected? In short, our focus of interest here is to understand how the mind handles the uncertainty caused by ambiguous figures and how people respond to them.

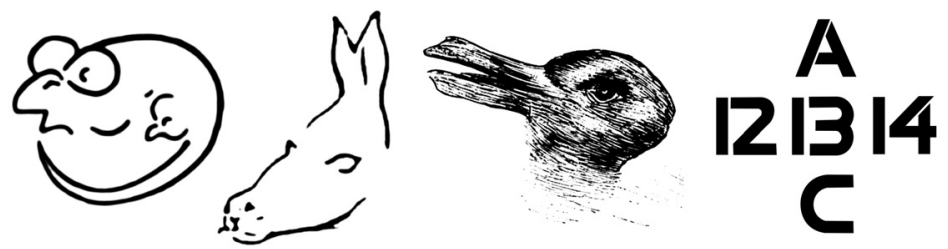

Figure 1. Four well-known ambiguous figures used in previous studies. From left to right: (1) face or mouse; (2) seal or donkey; (3) duck/rabbit (Jastrow); (4) number/letter. (Boring)

In the present study, we designed a two-alternative forced choice (2AFC) experiment in which an ambiguous picture (duck/rabbit) was shown rotated between $45^{\circ}$ and $225^{\circ}$ in steps of 11.25 degrees, with participants being asked to make categorization judgments. We hypothesized that the orientation would either facilitate or hinder the activation of a particular category. In the experiment, we manipulated the participants' 
response criterion by two set of instructions. Based on the results of this study we show that ambiguity perception of the duck-rabbit ambiguous figure is a categorization process determined not solely by the stimulus but also by mental observer's activity. Thus, prior experience, criterion for responding, demands of the task (instructions) and expectations can modify the rule used to categorize ambiguous pictures. The conditions in which every of these factors have more impact on the observer performance is discussed.

\section{Method}

\section{Participants}

Participants were 60 students from the University of Barcelona (44 female, 16 male), ranging in age from 18 to 27 years. They all had normal or corrected-to-normal visual acuity and provided written informed consent. The Declaration of Helsinki tenets of 1975 (as revised in October in 2008) were followed throughout the study, which received clearance by the Ethics Committee of the University of Barcelona.

\section{Stimuli}

The stimulus was a version of the famous duck-rabbit picture generated from an anonymous copy. This picture is an ambiguous (bistable) stimulus that was originally drawn by Joseph Jastrow (Jastrow, 1899; but see algo Brugger, 1999). Seventeen rotations of the duck-rabbit picture were used in the test, with the orientation ranging from $45^{\circ}$ to $225^{\circ}$ in steps of $11.25^{\circ}$ (see Figure 2).

The figures subtended a $3.43^{\circ} \times 2.86^{\circ}$ visual angle (height $\times$ width) on the participants' retina. A circle $\left(4.76^{\circ}\right.$ in radius) surrounding the figure was added to facilitate rotations. The stimuli were achromatic (black and white) at maximum contrast. Luminance of the background was $101.4 \mathrm{~cd} / \mathrm{m}^{2}$ and figure luminance was $0.08 \mathrm{~cd} / \mathrm{m}^{2}$.

\section{Apparatus}

The figures were presented on a 17-inch LCD screen with a spatial resolution of $1280 \times 768$ pixels and a refresh rate of $70 \mathrm{~Hz}$. The experiment was conducted in a room with four individual soundproofed small compartments. Each compartment was equipped with a PC (Intel Core i7) and a colour monitor placed on a shelf and a chair from which students could comfortably reach the keyboard and two single-button response boxes, one for the left and one for the right hand. The E-Prime 2.0 software was used to control stimulus presentation and to register the responses of the participants.

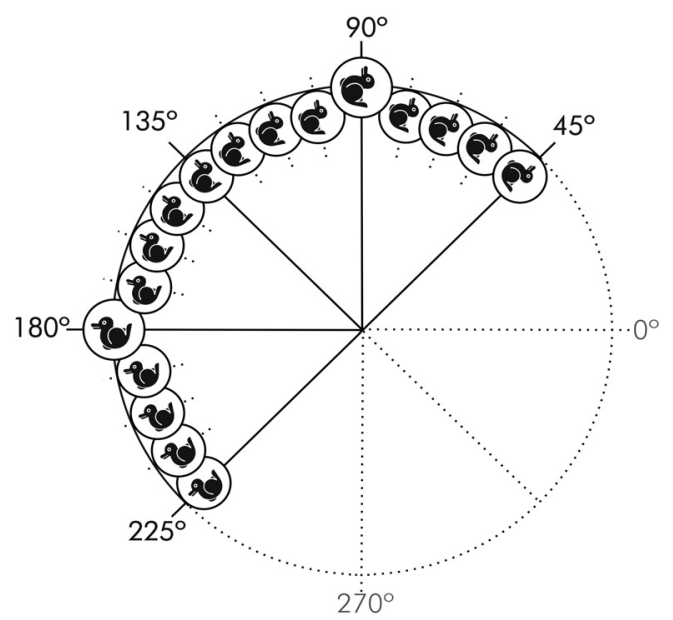

Figure 2. Seventeen rotations of the duck-rabbit ambiguous picture varying from $45^{\circ}$ to $225^{\circ}$ in steps of $11.25^{\circ}$ (people tend to see a rabbit in $90^{\circ}$ and a duck in $180^{\circ}$ rotation). 


\section{Procedure}

The experiment involved a single session and participants were tested in groups of four, each student in one compartment. Half of the participants performed the duck detection task (Group 1) and the others the rabbit detection task (Group 2). At the beginning of the experiment, students read specific instructions on the screen to become familiar with the task. They were informed that in the experiment, rabbits and ducks would appear on the screen (see Figure 3).

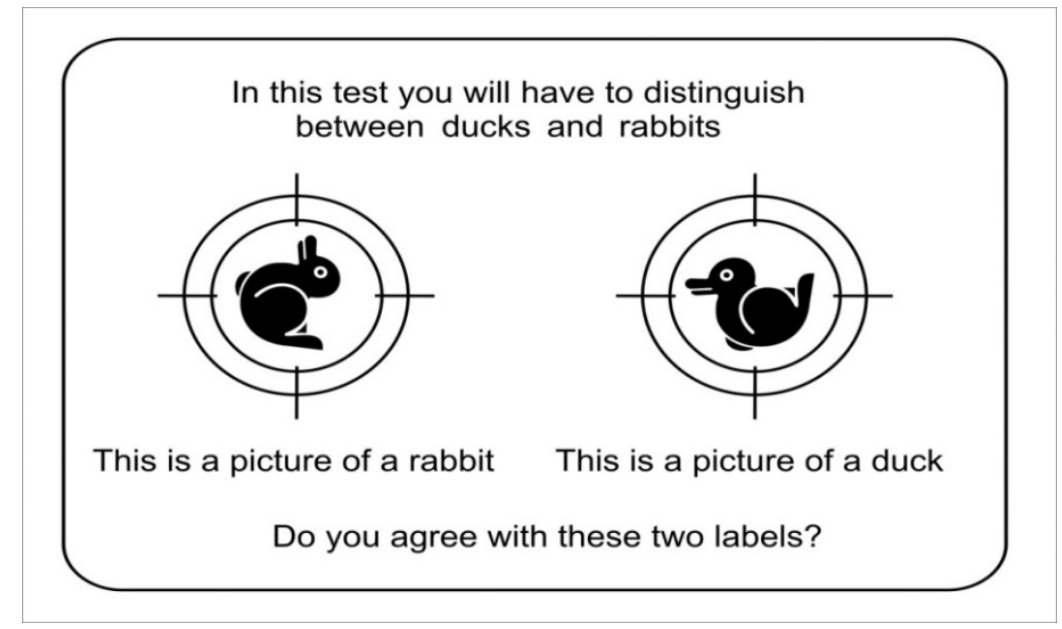

Figure 3. Screen in which participants received the first set of instructions and had to respond to the question.

The task was divided into two blocks of trials. The first and the second blocks were designed, respectively, to elicit "liberal" and "conservative" judgments from the participants.

In the first block, participants from Group 1 (duck detection) received the following instructions:

"In a nature reserve located in a nearby country, ducks and rabbits live alongside one another. A few hours ago an epidemic disease started to kill the ducks. In order to save them, ducks need to receive a vaccine. However, to prevent other diseases, rabbits must receive a certain amount of vitamin B. You are going to collaborate in a special mission in which you must shoot two kinds of darts. One contains the vaccine for ducks, which you can deliver by pressing the left button of the response device. For rabbits, you need to administer vitamin B by using the right response button".

We will refer to this instruction as "liberal" (using a loose criterion). In the same block, participants from Group 2 (rabbit detection) received similar instructions adapted to the rabbit. In total, 170 single pictures (17 rotations of the duck/rabbit picture, each repeated 10 times) were randomly presented for $200 \mathrm{~ms}$. Responses and reaction times (RT) of the participants were registered with E-prime.

After a minute's rest, new instructions were given for a second block of trials. For Group 1 (duck detection) the instructions were:

"Now we have to apply a second dose of vaccine and vitamin B to reinforce the effect. However, you must be very careful! If you administer the vaccine to the rabbits you will kill them. Likewise, if you administer the vitamin B to the ducks they will die. Thus, you cannot make any mistakes".

We will refer to this instruction as "conservative" (using a strict criterion). Analogously, we used a similar text adapted for Group 2 (rabbit detection). In this block, 170 pictures (17 rotations of the duck/rabbit picture, each repeated 10 times) were presented $(200 \mathrm{~ms})$ and responses were registered. Participants took around 30 minutes to complete the two blocks of trials. 


\section{Results}

\section{Descriptive Analysis of the Responses With Regard to Stimulus-Related Factors}

We computed the proportions of duck and rabbit categorizations in the 17 rotations of each block (stated by the instructions). Figure 4 shows the distribution of the proportions of target identifications for each criterion and the order in which the latter were applied. These plots reveal that each interpretation of the figure (seen "as a duck" or "as a rabbit") has an associated probability that varies as a function of the orientation of the figure. On the one hand, two prototypes can be clearly identified, one for each interpretation of the ambiguous figure (rabbit or duck), and located at the peak maximum of these functions. On the other hand, each orientation of the figure has a probability of assigning (or of perceiving) the oriented figure as one of the two interpretations. Finally, the junction of the two distributions (one for each target figure) of the proportion of responses reveals that the cut-off value separating them is located around $135^{\circ}$. Therefore, orientations near this value, the confusion zone, delimitate where uncertainty is greater. The distribution of responses also reveals the orientations in which the fuzzy boundaries of each prototype are located, namely $45^{\circ}$ and $225^{\circ}$ depending on the stimuli. In short, according to these distributions of responses (Figure 4) at least three different zones in uncertainty values can be defined: (1) around the prototypes $\left(90^{\circ} \pm 11.25^{\circ}\right.$ and $\left.180^{\circ} \pm 11.25^{\circ}\right)$, (2) near the extremes of the curves $\left(45^{\circ}+11.25^{\circ}\right.$ and $\left.225^{\circ}-11.25^{\circ}\right)$, and (3) around the cut-off $\left(135^{\circ} \pm 11.25^{\circ}\right)$.
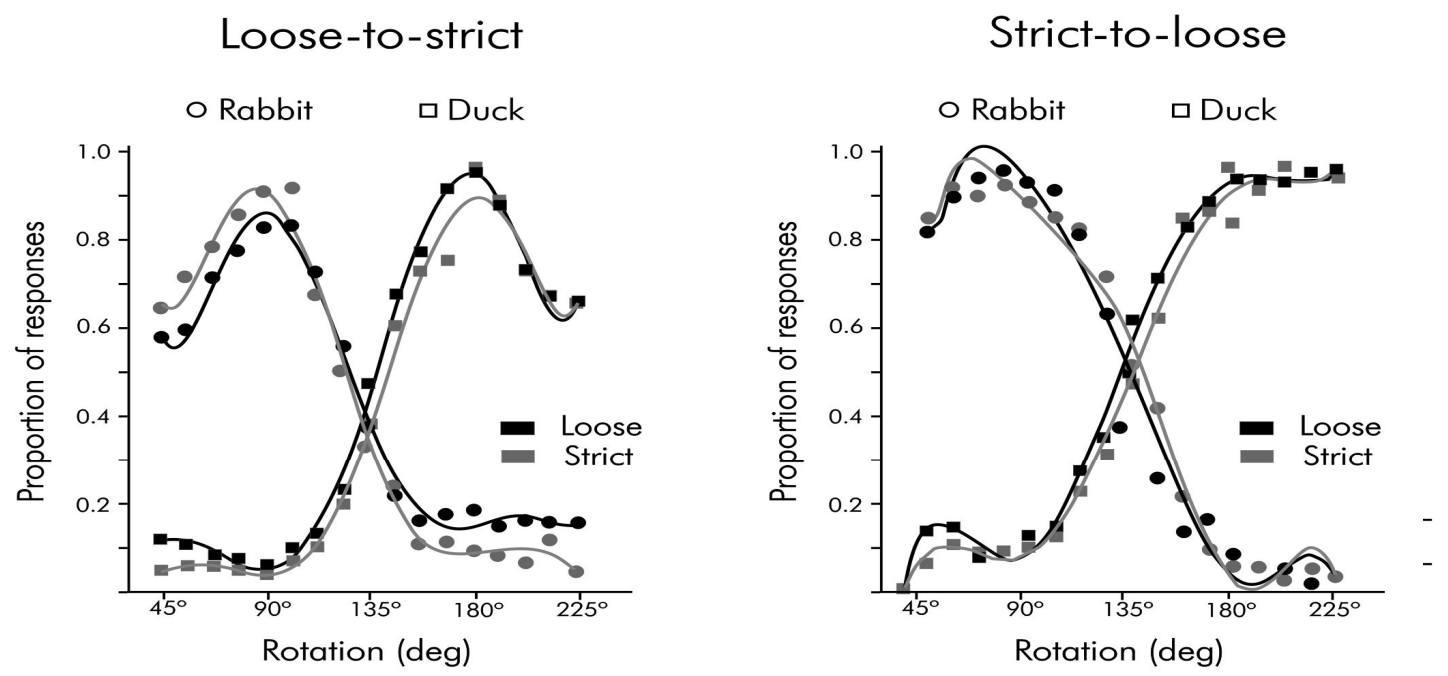

Figure 4. Distribution of the proportions of responses for each condition (set of instructions) of the ambiguous figure test as a function of the angle of rotation. Left panel: Loose-to-Strict criterion. Right panel: Strict-to-Loose criterion.

From these proportions of responses we examined the perceptual categories extending around the prototype in the range of $90^{\circ} \pm 45^{\circ}$ (i.e., between $45^{\circ}$ and $135^{\circ}$ ), which were labelled as a "rabbit", and around $180^{\circ} \pm 45^{\circ}$ (i.e., between $135^{\circ}$ and $225^{\circ}$ ), which were labelled as a "duck". We adjusted the two sets of proportions (duck and rabbit) to a Gaussian function and calculated two parameters of this model, namely the PSE (which must be related to the acceptability and accuracy of the prototype) and S.D. or the slope of the linearized function (which must be related to the sensitivity to discriminate).

Tables 1 and 2 show the location (PSE) and S.D. of the four psychometric functions, as well as the confidence intervals for the detection of ducks and rabbits in the two test conditions: "loose" or "strict". The 
locations of the two cumulative Gaussian functions (PSE) at the 0.5 probability of detection indicated the orientation corresponding to the prototype for the "rabbit" and "duck" categories. No significant differences were found with respect to the location of the psychometric function (PSE) when comparing the "loose" and "strict" criterion, as reflected by the confidence intervals. Note that in both categories the hypothesized orientation values of the prototypes $\left(90^{\circ}\right.$ and $\left.180^{\circ}\right)$ were underestimated (see Tables 1 and 2). Thus, according to this study the duck prototype is $178.45^{\circ}$, whereas the rabbit prototype is $81.92^{\circ}$. We then calculated the CE as the difference between the values of these prototypes (point of objective equality, i.e., the POE) and the PSE.

Interestingly, the CEs were statistically equivalent for rabbits and ducks. However, the order in which the criteria were applied produced a response bias. Thus, when the order was from a loose-to-strict criterion the CEs were higher for the "loose" criterion than for the "strict" one, and vice-versa for the order strict-to-loose criterion, where the CEs were higher for the "strict" criterion than for the "loose" one. In the latter case, participants tended to overestimate the value orientation of the prototype for the figure "duck", whereas they tended to underestimate the orientation for "rabbit".

Table 1

Parameters of the Cumulative Gaussian Functions that Best Fit the Data: Location (PSE) and Confidence Intervals of the Psychometric Functions of the Figures (Duck and Rabbit) and Conditions (C1 and C2). The Constant Error (CE) and Standard Error (SE) Associated With Data Collected When the Criterion Order Was Loose-to-Strict

\begin{tabular}{llccllll}
\hline & & PSE & CI (PSE) & S.D. & CI (S.D.) & CE & SE \\
\hline \multirow{2}{*}{ Rabbit } & Loose & 84.09 & $79.72-88.46$ & 0.064 & $0.055-0.073$ & +2.17 & 1.491 \\
& Strict & 80.71 & $77.68-83.73$ & 0.070 & $0.056-0.085$ & -1.21 & 1.543 \\
\multirow{2}{*}{ Duck } & Loose & 175.85 & $173.97-177.73$ & 0.067 & $0.051-0.082$ & -2.60 & 1.491 \\
& Strict & 178.57 & $175.99-181.14$ & 0.075 & $0.055-0.094$ & +0.12 & 1.543 \\
\hline
\end{tabular}

Table 2

Parameters of the Cumulative Gaussian Functions that Best Fit the Data: Location (PSE) and Confidence Intervals of the Psychometric Functions of the Figures (Duck and Rabbit) and Conditions (C1 and C2). The Constant Error (CE) and Standard Error (SE) Associated With Data Collected When the Criterion Order Was Strict-to-Loose

\begin{tabular}{llllllll}
\hline & & PSE & CI (PSE) & S.D. & CI (S.D.) & CE & SE \\
\hline \multirow{2}{*}{ Rabbit } & Loose & 80.17 & $77.16-83.19$ & 0.057 & $0.051-0.062$ & -1.75 & 1.491 \\
& Strict & 78.79 & $74.77-82.80$ & 0.054 & $0.049-0.059$ & -3.14 & 1.543 \\
\multirow{2}{*}{ Duck } & Loose & 178.85 & $177.28-180.41$ & 0.049 & $0.048-0.051$ & +0.40 & 1.491 \\
& Strict & 180.52 & $178.35-182.70$ & 0.051 & $0.049-0.053$ & +2.07 & 1.543 \\
\hline
\end{tabular}

Finally, the S.D. (slopes) of the psychometric functions were steeper when the participants applied first the strict criterion and then the loose one (see Table 2), as compared with vice-versa.

\section{Predicting the Observer's Responses}

In this section we analyze the influence of different factors on the response of the observer. Six discriminant analyses were conducted to predict whether an observer would respond yes or no to an ambiguous figure under different test conditions. The goal of these analyses was to find the best set of predictors of observers' responses, in other words, an exploratory attitude was adopted in order to identify the statistical weight with which a set of variables contribute to observers' responses under different levels of ambiguity of an 
ambiguous figure. These predictor variables could be used subsequently to guide theoretical study of how the mind handles uncertainty.

Note that the aim of this study was to determine the experimental test condition under which these factors (observer-related, stimulus-related and context-related) were used by participants to achieve better discrimination. Experimental conditions were defined by the target figure (duck or rabbit) and three groups of orientations of the ambiguous figure in which the set of orientations show a good fit to a linear function. These orientations were: $(1)$ around the rabbit and duck prototypes $\left(\approx 90^{\circ}: 90^{\circ} \pm 11.25^{\circ}\right.$ and $\left.\approx 180^{\circ}: 180^{\circ} \pm 11.25^{\circ}\right)$; (2) around $45^{\circ}\left(\approx 45: 45^{\circ}+11.25^{\circ}\right)$ and $225^{\circ}\left(\approx 225: 225^{\circ}-11.25^{\circ}\right)$; and $(3)$ around $135^{\circ}\left(\approx 135^{\circ}: 135^{\circ} \pm 11.25^{\circ}\right)$. Note that uncertainty increases from the first to the last of these orientations. Predictor variables were the "criterion" induced by the set of instructions (loose or strict), the "order" in which the instructions were given (loose-strict or strict-loose), the "orientation" of the ambiguous stimulus, the response given to the preceding trial (duck or rabbit) referred to as "prior response", the slope of the linearized Gaussian function best fitting the proportion of the observers' responses, and "PSE". Note, also, that the response given to the preceding trial ("prior response") constitutes previous experience and the set of instructions induces a "criterion" for responding in the tests, in other words, these variables are observer-related factors. By contrast, the "orientation" of the figure is a stimulus-related factor and the set of instructions ("criterion") given to observers is a contextual factor (mental set).

Table 3

Statistics of the Discriminant Analysis for all Examined Factors, According to the Different Experimental Test Conditions

\begin{tabular}{llllll}
\hline Figure & Orientations & Box's $M(p$-value $)$ & Eigen values & $\begin{array}{l}\text { Canonical } \\
\text { correlation }\end{array}$ & Wilks's $\lambda$ and Prob. \\
\hline \multirow{3}{*}{ Duck } & $\approx 90^{\circ} \& \approx 180^{\circ}$ & $973.43 ; p<0.001$ & 0.466 & 0.564 & $0.682 ; p<0.001$ \\
& $\approx 45^{\circ} \& \approx 225^{\circ}$ & $506.01 ; p<0.001$ & 0.101 & 0.303 & $0.908 ; p<0.001$ \\
& $\approx 135^{\circ}$ & $1620.93 ; p<0.001$ & 0.196 & 0.405 & $0.836 ; p<0.001$ \\
\hline \multirow{3}{*}{ Rabbit } & $\approx 90^{\circ} \& \approx 180^{\circ}$ & $785.65 ; p<0.001$ & 0.962 & 0.700 & $0.510 ; p<0.001$ \\
& $\approx 45^{\circ} \& \approx 225^{\circ}$ & $157.74 ; p<0.001$ & 0.166 & 0.377 & $0.858 ; p<0.001$ \\
& $\approx 135^{\circ}$ & $198.38 ; p<0.001$ & 0.209 & 0.416 & $0.827 ; p<0.001$ \\
\hline
\end{tabular}

When the target figure was "duck", Box's $M$ indicated that the assumption of equality of covariance matrices was violated for all the predictors on the dependent variable. However, given the large sample this problem was not regarded as serious. The discriminant function revealed a significant but low or moderate association between responses and all predictors (see values of the canonical correlation and Wilks' $\lambda$ in Table 3). Interestingly, the largest value (0.564) corresponds to the set of orientations around the duck and rabbit prototypes $\left(\approx 90^{\circ}\right.$ or $\approx 180^{\circ}$ ), accounting for $32 \%$ of the between-group variability. Accordingly, the largest Eigen value (0.466) was also observed for this set of orientations. However, closer analysis of the structure matrix (see values in Table 4) revealed that the discriminant loading (strength) of the different predictors varied with uncertainty, as defined by the set of orientations. Thus, for rotations around the prototype (i.e., $90^{\circ}$ and $\left.180^{\circ}\right)$ the best discriminant factor was clearly the true orientation of the figure (0.966); smaller loadings were obtained for the "S.D./slope" (-0.124) and the "prior response" (0.112), while "criterion", "order of the criteria" and PSE were poor predictors (see Figure 7 left). The cross-validated classification showed that overall 78.6\% of figures were correctly classified. For the set of orientations producing some uncertainty (i.e., $\approx 45^{\circ}$ and $\approx$ 
$\left.225^{\circ}\right)$ the best discriminant factors were the "criterion" (0.613), the "slope" $(-0.614)$ and the PSE $(-0.373)$, with "order of the criteria", "prior response" and the orientation of the figure being poor predictors (see Figure 7 centre). Note that the true physical orientation of the figure barely contributed to the prediction of response (-0.013). The cross-validated classification here showed that overall $79.1 \%$ of figures were correctly classified. Finally, for the set of orientations around the cut-off between the two interpretations (i.e., $\approx 135^{\circ}$ ) the best discriminant factors were the "slope" $(-0.758)$, the "orientation of the figure" $(0.453)$, the "prior response" (0.438) and the "order of the criteria" (0.371), with PSE and "criterion" as poor predictors (see Figure 7 right). Note that, similarly to the previous condition, observer variables (S.D. or slope and prior experience) as well as well as contextual variable (order of instructions) were relevant. The cross-validated classification here showed that overall $67.4 \%$ of figures were correctly classified, in other words, the model predictions were worse as uncertainty increased.

When the target figure was "rabbit", Box's $M$ indicated, as in the duck analysis, that the assumption of equality of covariance matrices was violated for all the predictors on the dependent variable. The discriminant function revealed a significant but moderate association between responses and all predictors, (see values of the canonical correlation and Wilks' $\lambda$ in Table 3$)$. Here again the largest value $(0.700)$ corresponds to the set of orientations around the duck and rabbit prototypes $\left(\approx 90^{\circ}\right.$ or $\left.\approx 180^{\circ}\right)$, accounting for $49 \%$ of between-group variability. Accordingly, the largest Eigen value (0.962) was also observed for this set of orientations. Closer analysis of the structure matrix (see Figure 7) revealed that, analogous to the duck categorization, the discriminant loading of the different predictors varied with uncertainty, as defined by the set of orientations. Thus, for rotations around the prototype (i.e., $90^{\circ}$ and $180^{\circ}$ ) the best discriminant factor was clearly the true physical orientation of the figure (0.969); a smaller loading was obtained for "prior response" (-0.115), while "criterion", "order of the criteria", "slope" and PSE were poor predictors (see Figure 5 left). The cross-validated classification showed that overall $85.9 \%$ of figures were correctly classified. For the set of orientations producing some uncertainty (i.e., $\approx 45^{\circ}$ and $\approx 225^{\circ}$ ) the best discriminant factors were PSE $(0.823$ ), that is, the implicit value of the prototype, and the "order of the criteria" $(0.790)$, whereas "orientation of the figure", the "prior response", "criterion" and "slope" were poor predictors (see Figure 5 centre). Note that, similarly to the "duck target", the true physical orientation of the figure contributed little to the prediction of response (-0.173). The cross-validated classification here showed that overall $74.6 \%$ of figures were correctly classified. Finally, for the set of orientations around the cut-off between the two interpretations (i.e., $\approx 135^{\circ}$ ) the best discriminant factors were the "prior response" $(-0.600)$, the "orientation of the figure" $(0.537)$ and PSE (0.356), although the "order of the criteria" $(0.278)$ and "slope" (-0.228) also had an appreciable influence; 'criterion' was a poor predictor (see Figure 5 right). Note that, similarly to the previous condition, observer variables (PSE, slope and prior experience) and a contextual variable (order of instructions) were relevant. The cross-validated classification here showed that overall $66.5 \%$ of figures were correctly classified, in other words, the model predictions were worse as uncertainty increased.

In brief, Figure 5 shows that ambiguity is related to uncertainty arising from the distance of a particular orientation to the prototypes. Thus, it reveals the experimental conditions under which stimulus-related, observer-related or contextual variables were preferably used. More specifically, it shows that participants based chiefly their responses on a stimulus related factor (i.e., the orientations of the figure) whether orientations were near to the prototypes $\left(\approx 90^{\circ}\right.$ and $\left.\approx 180^{\circ}\right)$. By contrast, for orientations of greater uncertainty $\left(\approx 45^{\circ}, 135^{\circ}\right.$ and $\left.\approx 225^{\circ}\right)$ the participants based chiefly their responses on observer's related factors (e.g., 
precision, prior experience, etc.) or contextual factors (e.g., criterion of response induced by the instructions). This analysis also reveals that there are notable differences between the two types of responses (duck or rabbit), which are not equally likely.
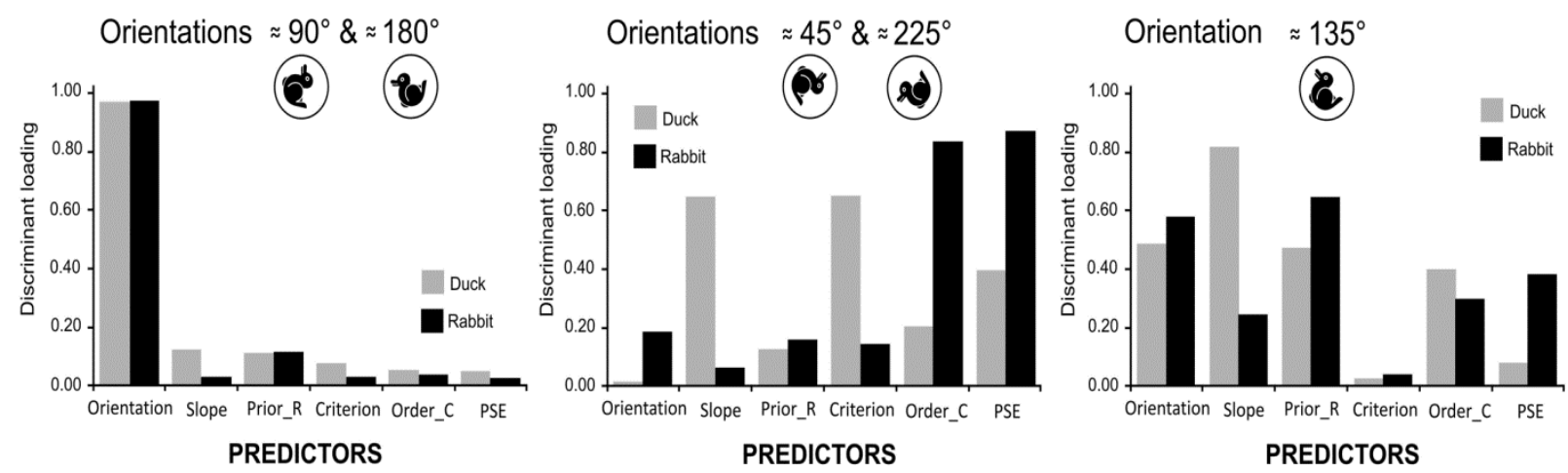

Figure 5. Distribution of the discriminant loadings (structural coefficients) for the different predictors used in the discriminant analysis according to the target figure and the three set of orientations: around the prototypes $\left(\approx 90^{\circ}\right.$ and $180^{\circ}$ ), orientations with high uncertainty $\left(\approx 45^{\circ}\right.$ and $\left.\approx 225^{\circ}\right)$, and orientation around the cut-off between the two interpretations $\left(\approx 135^{\circ}\right)$.

\section{Discussion}

In this study, we sought to ascertain how the mind handles the uncertainty caused by ambiguous figures and how people respond to them. In particular, we were interested in how observer-related, stimulus-related and contextual factors contribute to the observer's response, and in how these factors interact with one another.

Firstly, we evidenced that observers differentiated two categories, each one having a prototype linked to a particular orientation.

Data analysis revealed that participants were significantly more accurate in their categorization judgments when instructed to use a strict response criterion. However, this mainly occurred when they received 'strict' instructions first, rather than subsequent to "loose" instructions (i.e., an order effect was found).

The PSE of each psychometric function revealed that rotations corresponding to prototypes were underestimated in all categories (duck and rabbit) and conditions (instructions). In addition, the analysis of errors (constant error) showed that the two figures (duck and rabbit) were processed in a different way by observers. The two interpretations (duck or rabbit) did not have the same probability distribution of the response, having clear response biases. This response bias suggests that the direction of the bias may have to do with the more probable orientation of the discriminant feature for each category. In other words the duck's beak is usually horizontally oriented, while the rabbit's ears are usually vertically oriented (as suggested by the focal feature hypothesis). Thus the prototype for rabbits was at $82.6^{\circ}$ (in $\mathrm{C} 1$ ) and $80.3^{\circ}$ (in $\mathrm{C} 2$ ), while that for ducks was at $175.3^{\circ}$ and $177.2^{\circ}(\mathrm{C} 1$ and $\mathrm{C} 2$, respectively).

These results show that changes in orientation elicit changes in perception (retranslation of the features). In other words, the manipulation of a physical property (bottom-up processing) had some impact on the decision rule or criterion (top-down processing). In conclusion, our data show a bi-directional interaction between sensory- and cognitive-driven processes. This claim is in agreement with recent neuroimaging findings suggesting that frontal and parietal brain regions are involved in perceptual switching (de Graaf, de Jong, Goebel, van Ee, \& Sack, 2011; Knapen, Brascamp, Pearson, Ee, \& Blake, 2011; Windmann, Wehrmann, 
Calabrese, \& Güntürkün, 2006). Our results also provide support for the notion that the parietal lobe is involved in the processing of ambiguous figures. Physical rotation of figures (and also mental rotation, as shown by Shepard \& Metzler, 1971) does modify the orientation of features and can alter the interpretation of the figure. Thus, this psychophysical approach presents evidence that is convergent with neuroscientific research showing activity in the parietal lobe during the processing of oriented bistable stimuli (Britz, Landis, \& Michel, 2009; Kanai, Carmel, Bahrami, \& Rees, 2011).

As for the cut-off values used to dichotomize (categorize) the continuum of rotations between $90^{\circ}$ and $180^{\circ}$, results for the category "rabbit" showed that these values were similar (around $135^{\circ}$ ) in both conditions. This is one of the more interesting values to study because it is the value at which an interpretation switches to another. Therefore, factors responsible for this perceptual change could account for the perceptual alternation. We examined this point not from a psychophysics approach but rather by using discriminant analysis.

\section{How Stimulus Related, Observers and Context Factors Modulated Responses}

Results from exploratory discriminant analysis of six conditions (the result of combining two target figures and three set of orientations) help to clarify which variables contribute most to predicting the responses of observers. Thus, the structural coefficients of the factors included in the model revealed the experimental conditions under which stimulus-related, observer-related and contextual variables were preferably used. Specifically, Tables 3 together with the box plot in Figure 5, show that the variability in participants' responses is small for orientations near to the prototypes $(\approx 90$ and $\approx 180)$. By contrast, variability was larger for orientations with greater uncertainty associated $\left(\approx 45^{\circ}, 135^{\circ}\right.$ and $\left.\approx 225^{\circ}\right)$. This analysis also revealed that there are notable differences between the two types of responses (duck or rabbit), as far as the discriminant loadings are concerned.

The structural coefficients of the factors chiefly show how the discriminant loading of three kinds of variables were related with stimulus, observer and context depending on the uncertainty value that is promoted by the orientation. More specifically, we found that the observers' responses differed depending on the orientation of the ambiguous figure, varying from a minimum value (with low uncertainty) close to the prototypes to a maximum one (with high uncertainty) in those orientations more distant from the prototypes (i.e., $\approx 45^{\circ}$ and $\approx 225^{\circ}$ ). Thus, in this study, ambiguity can be characterized by uncertainty measured by the proportion of observers' responses to the ambiguous figure, as reflected in Figure 4, locating the tails of each distribution of responses in these orientations $\left(45^{\circ}\right.$ and $\left.225^{\circ}\right)$. Note that in previous studies the typical presentation of the duck/rabbit ambiguous figure has been $135^{\circ}$, precisely the location of the cut-off between the two interpretations (categories), overriding unwittingly those orientations in which uncertainty is middle or minimal. Furthermore, three different response biases were observed for different orientations with high uncertainty. Thus, the $45^{\circ}$ and $135^{\circ}$ orientations of the figure seem to promote a bias towards the response "rabbit", whereas the $225^{\circ}$ orientation seems to induce a bias in observers toward the response "duck".

\section{How Prior Experience Affected the Responses}

The exploratory discriminant analysis also revealed some influence of the previous response (or prior experience). Particularly, this factor had a determinant role in those cases where the figure achieved greater uncertainty $\left(\approx 45^{\circ}, 135^{\circ}\right.$ and $\left.\approx 225^{\circ}\right)$. Note that it is in these orientations where the typical features of the species (e.g., the duck's beak or the rabbit's ear) were not in the canonical orientation corresponding to any of the prototype, when in order to respond to the current trial, the response to the figure presented in the previous 
trial reached a high discriminant loading. Other studies (Bugelski \& Alampay, 1961; Goolkasian, 1987) have presented observers with an unambiguous version of an ambiguous figure (e.g., the old woman/young woman) and later showed the observers the ambiguous form. Results revealed that the most observers who have previously seen the unambiguous picture, for instance "as an old woman", see the ambiguous figure in the same way. Bugelski and Alampay (1961) conducted an experiment with the rat/man ambiguous figure, and showed one group of observers a set of animal pictures that did not include a rat. This top-down information had a powerful impact, since the number of observers who saw the ambiguous figure as a rat increased by as much as $80 \%$ in some experimental conditions. Leeper (1935) conducted a similar experiment but provided the top-down information in verbal form. He found that the group who heard a passage about rats before seeing the rat/man figure saw the picture as a rat twice as often as did the control group. However, there is also strong evidence for contextual influences on the perception of ambiguous figures (e.g., Goolkasian, 1987). Thus, it has been reported that children tested with Jastrow's duck/rabbit figure on Easter Sunday are more likely to see the figure as a rabbit, whereas if they are tested on a Sunday in October they tend to see it as a duck or similar bird (Brugger \& Brugger, 1993). In addition, it is known that briefly presenting an unambiguous version of a bistable figure prior to presenting the corresponding ambiguous figure can produce priming effects. In this regard, our results are consistent with the findings of Long et al. (1992), who showed the effects of short, prior presentations of an unambiguous version of an ambiguous figure (previous experience or perceptual learning) on the response of observers.

\section{How Instructions Influence the Response's Criterion}

With regard to the predictive power of the criterion induced by the set of instructions the structural coefficients of the exploratory discriminant analysis revealed that this factor only has a high discriminant loading in the set of orientations far from the prototype $\left(\approx 45^{\circ}\right.$ and $\left.\approx 225^{\circ}\right)$, or in the orientation located around the cut-off between the two interpretations $\left(135^{\circ}\right)$. By contrast, this factor had barely any influence on observers' responses when the orientation showed a good fit to the corresponding prototype $\left(90^{\circ}\right.$ or $\left.180^{\circ}\right)$. This result is in agreement with the Parasuraman (1986) thesis that any difference in the pattern of responses between "loose" and "strict" might be interpreted as evidence of higher-level influences on the categorization of ambiguous pictures.

\section{Conclusion}

From the results of this study we conclude that ambiguity perception of the duck-rabbit ambiguous figure is a categorization process that is not determined simply by the stimulus and its context but also by mental activity, i.e., we see with the mind as well as with our eyes. In sort, to solve an ambiguous figure is equivalent to decipher a message having implicit information, which is guided by a mental model: the prototype. To this goal the brain must create something like a cognitive equation in which a variety of information from different sources (stimuli, observer, context, etc.) should be integrated. Our study reveals how the coefficients of such an equation change as do the texting conditions.

\section{References}

Attneave, F. (1971). Multistability in perception. Scientific American, 225(6), 62-71. http://doi.org/10.1038/scientificamerican1271-62

Babich, S., \& Standing, L. (1981). Satiation effects with reversible figures. Perceptual and Motor Skills, 52(1), $203-210$. http://doi.org/10.2466/pms.1981.52.1.203 
Blakemore, C., \& Campbell, F. W. (1969). On the existence of neurones in the human visual system selectively sensitive to the orientation and size of retinal images. The Journal of Physiology, 203(1), 237-260.1.

Britz, J., Landis, T., \& Michel, C. M. (2009). Right parietal brain activity precedes perceptual alternation of bistable stimuli. Cerebral Cortex, 19(1), 55-65. http://doi.org/10.1093/cercor/bhn056

Brugger, P. (1999). One hundred years of an ambiguous figure: Happy birthday, duck/rabbit. Perceptual and Motor Skills, 89(3 Pt 1), 973-977. http://doi.org/10.2466/pms.1999.89.3.973

Brugger, P., \& Brugger, S. (1993). The Easter bunny in October: Is it disguised as a duck? Perceptual and Motor Skills, 76(2), 577-578. http://doi.org/10.2466/pms.1993.76.2.577

Bruner, J. S., \& Leigh, A. (1955). Perceptual identification and perceptual organization. Journal of General Psychology, 53, 21-28. http://doi.org/10.1080/00221309.1955.9710133

Boring, E. G. (1942). Sensation and perception in the history of experimental psychology. New York: Appleton Century.

Bugelski, B. R., \& Alampay, D. A. (1961). The role of frequency in developing perceptual sets. Canadian Journal of Psychology, $15,205-211$.

De Graaf, T. A., de Jong, M. C., Goebel, R., van Ee, R., \& Sack, A. T. (2011). On the functional relevance of frontal cortex for passive and voluntarily controlled bistable vision. Cerebral Cortex, 21(10), 2322-2331. http://doi.org/10.1093/cercor/bhr015

De Valois, K. K. (1977). Spatial frequency adaptation can enhance contrast sensitivity. Vision Research, 17(9), 1057-1065.

Ellis, S. R., \& Stark, L. (1978). Eye movements during the viewing of Necker cubes. Perception, 7(5), 575-581.

Flügel, J. C. (1913). The influence of attention in illusions of reversible perspective. British Journal of Psychology, 1904-1920, 5(4), 357-397. http://doi.org/10.1111/j.2044-8295.1913.tb00070.x

Gale, A. G., \& Findlay, J. M. (1983). Eye movement patterns in viewing ambiguous figures. Eye movements and psychological functions: International views, 145-168.

García-Pérez, M. A. (1989). Visual inhomogeneity and eye movements in multistable perception. Perception \& Psychophysics, 46(4), 397-400. http://doi.org/10.3758/BF03204995

Goolkasian, P. (1987). Ambiguous figures: Role of context and critical features. The Journal of General Psychology, 114(3), 217-228.

Goolkasian, P., \& Woodberry, C. (2010). Priming effects with ambiguous figures. Attention, Perception, \& Psychophysics, 72(1), 168-178. http://doi.org/10.3758/APP.72.1.168

Gregory, R. L. (1970). The intelligent eye. New York: McGraw-Hill.

Hochberg, J. E. (1950). Figure-ground reversal as a function of visual satiation. Journal of Experimental Psychology, $40(5), 682$.

Jastrow, J. (1899). The mind's eye. Popular Science Monthly, 299-312.

Kanai, R., Carmel, D., Bahrami, B., \& Rees, G. (2011). Structural and functional fractionation of right superior parietal cortex in bistable perception. Current Biology, 21(3), R106-R107. http://doi.org/10.1016/j.cub.2010.12.009

Knapen, T., Brascamp, J., Pearson, J., Ee, R. van, \& Blake, R. (2011). The role of frontal and parietal brain areas in bistable perception. The Journal of Neuroscience, 31(28), 10293-10301. http://doi.org/10.1523/JNEUROSCI.1727-11.2011

Kornmeier, J., \& Bach, M. (2004). Early neural activity in Necker-cube reversal: Evidence for low-level processing of a gestalt phenomenon. Psychophysiology, 41(1), 1-8. http://doi.org/10.1046/j.1469-8986.2003.00126.x

Kornmeier, J., \& Bach, M. (2012). Ambiguous figures-What happens in the brain when perception changes but not the stimulus. Frontiers in Human Neuroscience, 6, 51. http://doi.org/10.3389/fnhum.2012.00051

Leeper, R. (1935). A study of a neglected portion of the field of learning-The development of sensory organization. The Pedagogical Seminary and Journal of Genetic Psychology, 46(1), 41-75. http://doi.org/10.1080/08856559.1935.10533144

Leopold, D. A., \& Logothetis, N. K. (1999). Multistable phenomena: Changing views in perception. Trends in Cognitive Sciences, 3(7), 254-264.

Long, G. M., \& Toppino, T. C. (2004). Enduring interest in perceptual ambiguity: Alternating views of reversible figures. Psychological Bulletin, 130(5), 748-768. http://doi.org/10.1037/0033-2909.130.5.748

Long, G. M., Toppino, T. C., \& Mondin, G. W. (1992). Prime time: Fatigue and set effects in the perception of reversible figures. Perception \& Psychophysics, 52(6), 609-616. http://doi.org/10.3758/BF03211697

Maffei, L., Fiorentini, A., \& Bisti, S. (1973). Neural correlate of perceptual adaptation to gratings. Science (New York, N.Y.), 182(4116), 1036-1038.

Mathes, B., Strüber, D., Stadler, M. A., \& Basar-Eroglu, C. (2006). Voluntary control of Necker cube reversals modulates the EEG delta- and gamma-band response. Neuroscience Letters, 402(1-2), 145-149. http://doi.org/10.1016/j.neulet.2006.03.063 
Parasuraman, R. (1986). Vigilance, monitoring, and search. In K. R. Boff, L. Kaufman, \& J. P. Thomas (Orgs.), Handbook of perception and human performance, Vol. 2: Cognitive processes and performance (pp. 1-39). Oxford, England: John Wiley $\&$ Sons.

Posner, M. I. (1980). Orienting of attention. The Quarterly Journal of Experimental Psychology, 32(1), 3-25.

Rock, I., Hall, S., \& Davis, J. (1994). Why do ambiguous figures reverse? Acta Psychologica, 87(1), 33-59.

Rock, I., \& Mitchener, K. (1992). Further evidence of failure of reversal of ambiguous figures by uninformed subjects. Perception, 21(1), 39-45.

Shepard, R. N., \& Metzler, J. (1971). Mental rotation of three-dimensional objects. Science (New York, N.Y.), 171(3972), 701-703.

Toppino, T. C. (2003). Reversible-figure perception: Mechanisms of intentional control. Perception \& Psychophysics, 65(8), 1285-1295.

Toppino, T. C., \& Long, G. M. (2005). Top-down and bottom-up processes in the perception of reversible figures: Toward a hybrid model. In N. Ohta, C. M. MacLeod, \& B. Uttl (Orgs.), Dynamic Cognitive Processes (pp. 37-58). Springer Tokyo.

Tsal, Y. (1994). Effects of attention on perception of features and figural organisation. Perception, 23(4), 441-452.

Windmann, S., Wehrmann, M., Calabrese, P., \& Güntürkün, O. (2006). Role of the prefrontal cortex in attentional control over bistable vision. Journal of Cognitive Neuroscience, 18(3), 456-471. http://doi.org/10.1162/089892906775990570

Zeki, S. (2004). The neurology of ambiguity. Consciousness and Cognition, 13(1), $173-196$. http://doi.org/10.1016/j.concog.2003.10.003 